\title{
Thermal Performance of Heat Shield Under High-Temperature Jet
}

\author{
Zheng Hu${ }^{1}$, Jiajia Sui ${ }^{1}$, Chengbin Zhang ${ }^{1}$ \\ ${ }^{1}$ Key Laboratory of Energy Thermal Conversion and Control of Ministry of Education \\ School of Energy and Environment, Southeast University \\ Nanjing, Jiangsu 210096, PR China \\ 213153537@seu.edu.cn; 854739739@qq.com; cbzhang@seu.edu.cn
}

\section{Extended Abstract}

Under the high-temperature jet, the thermal performance of conventional heat shield only relies on the heat conduction of the metal plate, leading to the unpreferrable local high temperature. How to reduce the local high temperature of heating surface for the heat shield, is a key issue to be considered in the design of heat shield. This paper proposes a new type heat shield, in which a new composite phase change material is adopted as the base material. In order to guide the design of heat shield, a two-dimensional model of unsteady heat transfer in the heat shield is developed and numerically analyzed to investigate thermal performance of heat shield under high-temperature jet. In the model, the hot side of heat shield is under the interaction of high-speed inclined jet in large space while the cold side is cooled by the water. Through this mathematical model, the thermal response performance of heat shield with phase change material is presented and compared with corresponding conventional metal plate. The thermal response performance is characterized by the maximum surface temperature of hot side and dynamic temperature variation. The effect of flow rate and jet velocity on the thermal response performance is examined and analyzed.

The results indicate that, the maximum surface temperature of hot side for heat shield is effectively reduced by using the composite phase change material as compared with conventional aluminum heat shield. When the cooling water flow rate is 1 ton/h, the maximum surface temperature of hot side for heat shield with phase change material is $100 \mathrm{k}$ smaller than conventional aluminum heat shield under the interaction of high temperature jet. The cooling water flow rate has a great influence on the thermal recovery performance of heat shield which has little effect on the maximum surface temperature of hot side under high-temperature jet. The increase in water flow rate significantly reduces the water outlet temperature. The water flow rate should be reasonably selected in the design and development of heat shield to ensure that the water outlet is at a reasonable temperature level. 